factors and barriers toward the development of future interventions to improve functional outcomes. The second presentation will describe the contribution of organizational factors such as staff skill mix and patients' complexity to hospitalization outcomes, demonstrating how functional status of all hospitalized patients at the unit is related to individual outcomes. The following presentation will focus on a rarely described topic, disruption of patients' routine during hospitalization and its adverse effect on hospitalization outcomes. Recommendations to take into consideration patients' personal routine into interventions aimed at improving hospitalization outcomes will be proposed. The fourth presentation will discuss the dose-response effect of mobility on functional decline. This presentation will present a suggested cut-point for in-hospital step count. Finally, we will introduce the Walk-FOR intervention, developed in accordance with the above mentioned processes aimed to improve hospitalization outcomes.

\section{HOSPITAL ACQUIRED FUNCTIONAL DECLINE: THE ROLE OF IN-HOSPITAL PROCESSES BEYOND PERSONAL RISK FACTORS}

A. Zisberg ${ }^{1}$, G. Sinoff ${ }^{2}$, N. Gur-Yaish ${ }^{3}$, O. Tonkikh ${ }^{1}$, E. Shadmi ${ }^{1}, 1$. The Cheryl Spencer Department of Nursing Faculty of Social Welfare and Health Science, University of Haifa, Israel, Haifa, Israel, 2. Department of Gerontology, Faculty of Social Welfare and Health Sciences, University of Haifa, Israel, Haifa, Israel, 3. Center for Research and Study of Aging, Faculty of Social Welfare and Health Sciences, University of Haifa, Israel, Haifa, Israel

To test a comprehensive model accounting for individual risk factors and hospitalization processes that lead to functional decline (FD) at discharge and one-month follow-up, a prospective cohort study was conducted in two medical centers. A total of 684 participants $(70+)$ interviewed at-admission, during hospitalization, at-discharge and one-month follow-up. In-hospital mobility, continence care, sedativehypnotic consumption, satisfaction with the hospital environment and nutrition consumption were assessed to model FD from pre-morbid to discharge and one-month post-discharge. In-hospital mobility $(\beta=-.48, \mathrm{p}<.001)$ and continence care $(\beta=-.12, p<.001)$ were the most dominant process factors directly related to FD at discharge and one-month post discharge, together with personal risk factors accounted for $64 \%$ and $32 \%$ of the variance, respectively. In-hospital mobility and continence care were inter-correlated $(\mathrm{p}=.49$, $\mathrm{p}<.001)$ proposing shared risk factors and need to address them simultaneously in future interventions. These are potentially modifiable hospitalization risk factors for which practice and policy should be targeted.

\section{TEAM SKILL-MIX AND PATIENT CASE-MIX: ARE THEY RELATED TO HOSPITALIZATION FUNCTIONAL OUTCOMES?}

E. Shadmi ${ }^{3}$, O. Tonkikh ${ }^{3}$, G. Sinoff ${ }^{1}$, Z. Oleg 2 , A. Zisberg ${ }^{3}$, 1. Department of Gerontology, Faculty of Social Welfare and Health Sciences, University of Haifa, Israel, Haifa, Israel, 2. University of Washington, Seattle, Washington, 3. The Cheryl Spencer Department of Nursing, Faculty of Social Welfare and Health Science, University of Haifa, Israel, Haifa, Israel, Israel
We aimed to test the association between nursing skillmix and patient case-mix on the functioning of older adults hospitalized in internal medicine wards of general hospitals. We performed a retrospective study of 463 participants of the HoPE-FOR study. The number of nurses/ patients and the percent of patients with low functioning of all patients at the unit was assessed for the duration of hospitalization for each participant. Survey data was used to elicit information on participants' functioning and personal risk factors. A total of $224(48.3 \%)$ patients experienced FD. Number of patients at the unit with low functioning and the number of nurses was positively correlated $\left(r_{p}=0.69, p<0.001\right)$. Percent of unit patients who had low levels of functioning was associated with increased odds of FD of study participants $(\mathrm{OR}=1.60,95 \% \mathrm{CI}=1.18-$ 2.16), controlling for all known risk factors. We conclude that nursing skill-mix should account for the functioning levels of hospitalized patients.

\section{OLDER ADULTS' PERSONAL ROUTINE AT TIME OF HOSPITALIZATION}

N. Gur-Yaish ${ }^{1}$, A. Zisberg ${ }^{2}$, 1. Center for Research and Study of Aging, Faculty of Social Welfare and Health Sciences, University of Haifa, Israel, Haifa, Israel, 2. The Cheryl Spencer Department of Nursing Faculty of Social Welfare and Health Science, University of Haifa, Israel, Haifa, Israel

This study is the first to explore whether hospitalization disrupts the daily routines of dependent and independent older adults. Data were collected as part of a prospectively designed study from 330 hospitalized older adults age $70+$. Patients reported prehospitalization frequency, duration, and timing of basic activities of daily living and leisure activities at hospital admission. Hospital routine was assessed on day of discharge. Results indicated that frequency and duration of most activities decreased during hospitalization; the sharpest decrease was in getting dressed. Showering occurred two hours earlier in the hospital, and getting dressed an hour and a half later. For dependent respondents, the greatest change was in duration; for independent respondents, the greatest change was in frequency. Given the importance of personal routine maintenance to health and well-being, it should be considered a key component for intervention to minimize the adverse functional outcomes and impact recovery.

\section{“NO ONE SIZE FITS ALL": DEVELOPING A SITE-SPECIFIC, IN-HOSPITAL MOBILITY INTERVENTION-THE WALK FOR®}

M. Agmon ${ }^{1}$, E. Gili2,3, A. Zisberg ${ }^{1}$, N. Gur-Yaish ${ }^{4}, 1$. The Cheryl Spencer Department of Nursing Faculty of Social Welfare and Health Science, University of Haifa, Israel, Haifa, Israel, 2. Bnei- Zion Medical Center, Haifa, Haifa, Israel, 3. Faculty of Medicine, Technion, Haifa, Haifa, Israel, 4. Center for Research and Study of Aging, Faculty of Social Welfare and Health Sciences, University of Haifa, Israel, Haifa, Israel

The few protocols aimed to improve in- hospital mobility require further work to response to site-specific needs and available resources. The presentation will describe a process of developing a site-tailored mobility intervention 